\title{
Pushing the Limits on SEM Quantification - Combined Quantification with SDD and Fully Focussing WD detectors
}

\author{
C.L. Collins ${ }^{1}$, J. Holland ${ }^{1}$, S.R. Burgess ${ }^{1}$ \\ ${ }^{1}$ Oxford Instruments Nanoanalysis, Halifax Road, High Wycombe, HP12 3SE, UK
}

Wavelength Dispersive Spectroscopy (WDS) was, until recently, the only way to achieve accurate, repeatable minor $(<1.0 \mathrm{wt} \%)$ and trace $(<0.1 \%)$ element analysis on the SEM. As a single spectrometer solution, however, it is limited by serial data acquisition and the need for high probe currents.

Improvements in detector resolution and quantification software means that Energy Dispersive Spectroscopy (EDS) is now producing useful results in what was previously WD territory (i.e. overlap corrections and the quantification of minor elements with concentrations below $1 \%$ ). Consequently, multi-element analysis with a single WDS spectrometer is becoming less likely, but overall, it still remains the best technique on the SEM for single element analysis, mapping and separating overlaps and measuring concentration at trace level.

The combination of WDS with EDS utilises the strengths of both techniques offering simultaneous fast and accurate quantification down to less than 100ppm. The faster EDS is used for major elements while the minor and trace element analysis is done by WDS.

Here we present results showing how combined ED-WD mapping and trace element quantification can be achieved even at high beam currents ( $>20 \mathrm{nA}$ ), without any loss in accuracy of results. By introducing a variable collimator on the EDS detector (the Oxford Instruments X-Max+) simultaneous high beam current ED-WD acquisition is possible.

Figure 1 shows the combined ED-WD quantification data from a verified glass standard (SRM 621). It contains both light and heavy elements at a variety of concentrations down to 80ppm of Ti. There is also the potential issue of overlaps in the sample between $\mathrm{Ba}$ and $\mathrm{Ti}$, separated by $42 \mathrm{eV}$. At these low concentration levels, this overlap would be extremely difficult, if not impossible, to solve with EDS alone.

The data was collected using an Oxford Instruments Wave spectrometer at $20 \mathrm{kV}$ accelerating voltage and 20nA beam current in conjunction with an Oxford Instruments X-Max ${ }^{\mathrm{N}} 150 \mathrm{~mm}^{2}$ EDS detector fitted with an X-Max+. Higher beam currents can decrease WD analysis times, but must be balanced against damaging the sample. Minor and trace elements ( $\mathrm{Mg}, \mathrm{S}, \mathrm{Ti}, \mathrm{Fe}$ and As) were measured with WDS while all remaining elements were analysed with EDS. Five results were averaged and compared to the standard. In all cases, the calculated WD average was within 2 sigma of the published standard concentrations. At 20nA, there was evidence of Na migration within the glass.

Figure 2 shows X-ray maps collected at 100nA on an experimental slag sample. In this sample the K lines from important elements ( $\mathrm{Mn}, \mathrm{Fe}$ and $\mathrm{Cr}$ ) overlap. Without any further software correction, the pure intensity (windows integral) maps fail to separate the $\mathrm{Mn} \mathrm{K} \alpha$ and $\mathrm{Cr} \mathrm{K} \beta$ contributions, resulting in a very misleading $\mathrm{Mn} \mathrm{K \alpha}$ window map. When AZtec EDS TruMap is used to deconvolute the 
spectra at each pixel, the overlap is corrected successfully, but the data is very noisy and difficult to interpret. The WDS map has much less noise and provides an accurate representation of the Mn elemental distribution in this sample.

\section{Conclusions}

Improvements in EDS hardware and software, and the introduction of large area SDD detectors mean that EDS detectors are beginning to rival WDS for results when it comes to the quantification of multi element samples, even for minor elements. For individual elements (i.e. trace element $(<0.1$ wt\%) quantification) and mapping in the presence of major elemental overlaps, however, WDS continues to offer the most accurate results and sensitive detection. Combined ED-WD analysis offers analysts the best combination, both in speed and accuracy and analysing the whole elemental range right down to less than 100ppm.

\begin{tabular}{|c|c|c|c|c|c|c|c|c|c|c|c|c|c|}
\hline & 0 & $\mathrm{Na}$ & Mg & Al & $\mathrm{Si}$ & $\mathbf{S}$ & $\mathbf{K}$ & $\mathrm{Ca}$ & $\mathrm{Ti}$ & $\mathrm{Fe}$ & As & $\mathrm{Ba}$ & \\
\hline Method & EDS & EDS & WDS & EDS & EDS & WDS & EDS & EDS & WDS & WDS & WDS & WDS & TOTAL \\
\hline Spectrum 1 & 46.0600 & 8.9824 & 0.1546 & 1.4845 & 33.1345 & 0.0375 & 1.7571 & 7.7543 & 0.0059 & 0.0436 & 0.0282 & 0.1122 & 99.5549 \\
\hline Spectrum 2 & 46.1162 & 8.8870 & 0.1531 & 1.4840 & 33.0679 & 0.0408 & 1.7715 & 7.7198 & 0.0040 & 0.0332 & 0.0162 & 0.1044 & 99.3980 \\
\hline Spectrum 3 & 45.9721 & 8.8007 & 0.1533 & 1.4459 & 33.0455 & 0.0403 & 1.7964 & 7.7463 & 0.0082 & 0.0220 & 0.0319 & 0.1068 & 99.1695 \\
\hline Spectrum 4 & 45.7567 & 8.8505 & 0.1553 & 1.5287 & 33.0079 & 0.0385 & 1.7230 & 7.7468 & 0.0033 & 0.0305 & 0.0253 & 0.1104 & 98.9768 \\
\hline Spectrum 5 & 46.1661 & 8.8001 & 0.1551 & 1.4760 & 33.0792 & 0.0460 & 1.7191 & 7.7406 & 0.0107 & 0.0381 & 0.0191 & 0.0845 & 99.3346 \\
\hline AVERAGE: & 46.0142 & 8.8641 & 0.1543 & 1.4838 & 33.0670 & 0.0406 & 1.7534 & 7.7416 & 0.0064 & 0.0335 & 0.0241 & 0.1037 & 99.2868 \\
\hline Sigma & 0.0189 & 0.0530 & 0.0050 & 0.0240 & 0.0670 & 0.0030 & 0.0240 & 0.0390 & 0.0030 & 0.0060 & 0.0040 & 0.0150 & \\
\hline STANDARD & 46.1000 & 9.4400 & 0.1600 & 1.4600 & 33.2200 & 0.0500 & 1.6700 & 7.6400 & 0.0080 & 0.0300 & 0.0200 & 0.1100 & 99.9080 \\
\hline
\end{tabular}

Figure 1 Combined ED-WD quantification results from a glass standard (SRM 621). The minor and trace elements were quantified by WDS and all others by EDS. The combined total measurement time was 24 minutes.

(a)

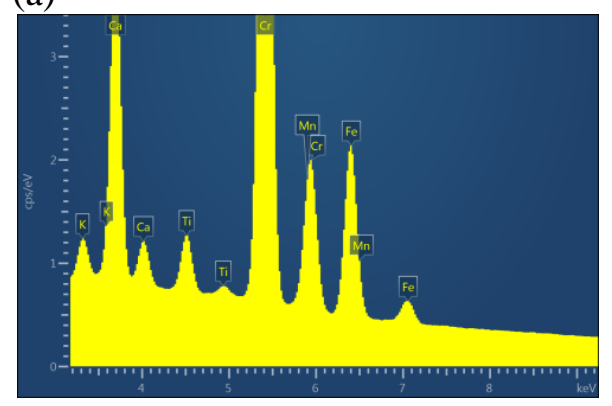

(b)

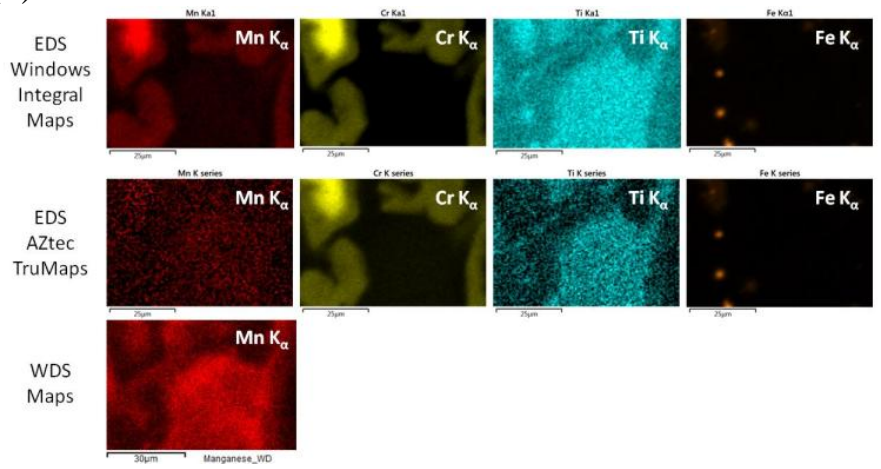

Figure 2 High beam current (100nA) mapping of a experimental slag sample. (a) Spectrum showing the spectral overlap between $\operatorname{CrK} \beta$ and $\operatorname{MnK} \alpha$ (b) Windows integral, TruMaps and WDS maps collected from the sample. 